\title{
IgG4-Unrelated Type 1 Auto Immune Pancreatitis Manifesting with Pancreatic Head Mass Mimicking Cancer
}

\author{
Adnane Arrami1 ${ }^{*}$, Nabil Moatassim Billah'², Tarik Addioui ${ }^{3}$, Ittimad Nassar ${ }^{2}$ \\ ${ }^{1}$ Radiology Department, Military Hospital, Mohammed V University, Rabat, Morocco \\ ${ }^{2}$ Central Radiology, Ibn Sina Hospital, Mohammed V University, Rabat, Morocco \\ ${ }^{3}$ Gastroenterology Department, Military Hospital, Mohammed V University, Rabat, Morocco \\ Email: *adnane.arrami@gmail.com
}

How to cite this paper: Arrami, A., Billah, N.M., Addioui, T. and Nassar, I. (2018) IgG4-Unrelated Type 1 Auto Immune Pancreatitis Manifesting with Pancreatic Head Mass Mimicking Cancer. Open Journal of Clinical Diagnostics, 8, 7-15. https://doi.org/10.4236/ojcd.2018.82002

Received: May 7, 2018

Accepted: June 25, 2018

Published: June 28, 2018

Copyright $\odot 2018$ by authors and Scientific Research Publishing Inc. This work is licensed under the Creative Commons Attribution International License (CC BY 4.0).

http://creativecommons.org/licenses/by/4.0/

\begin{abstract}
Auto immune pancreatitis (AIP) is an uncommon form of chronic pancreatitis that has been divided into type 1 and type 2 which have distinct histopathology and clinical features. Type 1 AIP seems to be the pancreatic manifestation of an IgG4-related systemic disease, characterized by elevated IgG4 serum levels, infiltration of IgG4-positive plasma cells and extrapancreatic lesions. When manifesting as a focal disease in the pancreas, it can be challenging to differentiate it from pancreatic cancer (PC). Because AIP is typically responsive to steroid therapy without the need for resection, differentiation between these two diseases is critical. We report the case of a 56-year-old man with initial suspect of PC, and final diagnosis of type 1 AIP according to the International Consensus Diagnostic Criteria (ICDC) without elevated levels of serum IgG4 or need for a histology sample. We take a review of literature in order to improve the diagnostic accuracy of AIP and describe clinical and imaging features to differentiate it from PC and avoid unnecessary surgery due to misdiagnosis.
\end{abstract}

\section{Keywords}

Autoimmune Pancreatitis (AIP), Pancreatic Head Mass, Pancreatic Cancer (PC), Ig4 Unrelated, Imaging Features

\section{Introduction}

Autoimmune pancreatitis (AIP) is a rare pancreatic disorder among chronic pancreatitis that may mimic pancreatic cancer (PC) [1]. It was first characterized in 1961 from a patient showing signs of pancreatic damage with associated au- 
toimmunity. The term AIP was first used in 1995 by Yoshida et al. to describe a type of chronic pancreatitis associated with a Sjogren-like syndrome [2]. In 2002, the Japan Pancreas Society (JPS) proposed the world's first clinical diagnostic criteria for AIP (JPS 2002) [3], which was revised in 2006 (JPS, 2006). Subsequently, the International Consensus Diagnostic Criteria (ICDC) was proposed in 2011 and AIP was divided into type 1 and type 2 with distinct histopathology and clinical features [2] [3]. Type 1 is more frequent than type 2 and shares some clinical, biological and radiological features with PC making the differential diagnosis very difficult [1]. We report the case of a 56-year-old man with initial suspect of PC, and final diagnosis of type 1 AIP according to the ICDC without elevated levels of serum IgG4 or need for a histology sample. The aim is to describe clinical and imaging features of AIP to differentiate it from PC and avoid unnecessary surgery due to misdiagnosis.

\section{Case Report}

A 56-year-old male was admitted to emergency department with 2 weeks history of sub acute upper abdominal pain. He has medical past history of alcohol and tobacco abuse since 30 years without other illness or family history. He also reports a weigh loss of $4 \mathrm{Kg}$ in 2 months. The Physical examination revealed a slight jaundice without fever. Abdominal ultrasonography demonstrated a 60 $\mathrm{cm}$-sized hypoechoic mass in the pancreatic (Figure 1) without dilation of the intrahepatic and extrahepatic bile ducts and the main pancreatic duct. Laboratory tests showed: elevated levels of alkaline phosphatase (ALP: 184 IU/L; normal range, 45 - $125 \mathrm{IU} / \mathrm{L}$ ), ) and $\gamma$-glutamyltransferase ( $\gamma$-GT: $118 \mathrm{IU} / \mathrm{L}$; normal range, 10 - $60 \mathrm{IU} / \mathrm{L}$ ), normal levels of total bilirubin, aspartate aminotransferase and alanine aminotransferase, and mild elevated level of lipase (92 IU/L; normal range, 6-48 IU/L). Test of tumor markers revealed mild elevated level of CA199 (52 IU/ml; normal range, 0 - $37 \mathrm{IU} / \mathrm{ml}$ ).Complete blood count and renal function were normal. Pancreatic cancer was suspected and the patient was referred to the gastroenterology department for additional management. Abdominal computed tomography (CT) revealed: localized swelling of the head of the pancreas that shows fair enhancement resulting almost isodense in the delayed phase with a hypodense peripancreatic Rim slightly enhanced in the delayed phase, and mild dilation of common bile duct $(8 \mathrm{~mm})$ with a thickened enhanced wall without dilation of the main pancreatic duct (Figure 2). There were neither calcifications nor distal pancreatic atrophy. Regional limphnodes and liver metastases were absent. At magnetic resonance image (MRI), the affected portion of the pancreas was hypointense on T1-weighted fat-saturated images and hyperintense on T2-weighted fat-saturated images, with diffusion coefficient restriction. At dynamic examination the pancreatic lesion showed heterogeneous diminished enhancement in the early phase and delayed enhancement in the late phase of contrast. The capsule-like rim described at CT was hypointense on both T1 and T2-weighted images, and has delayed moderate enhancement on 


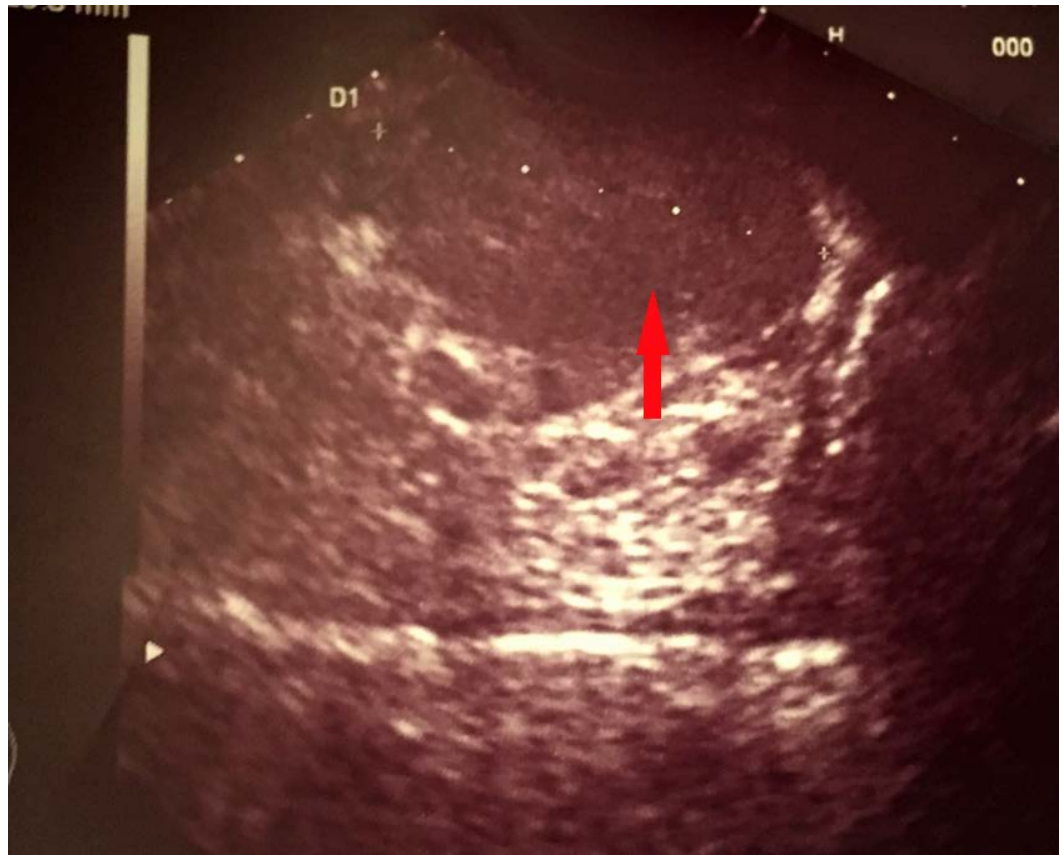

Figure 1. Transversal abdominal ultrasonography: hypoechoic mass in the pancreatic (red arrow).
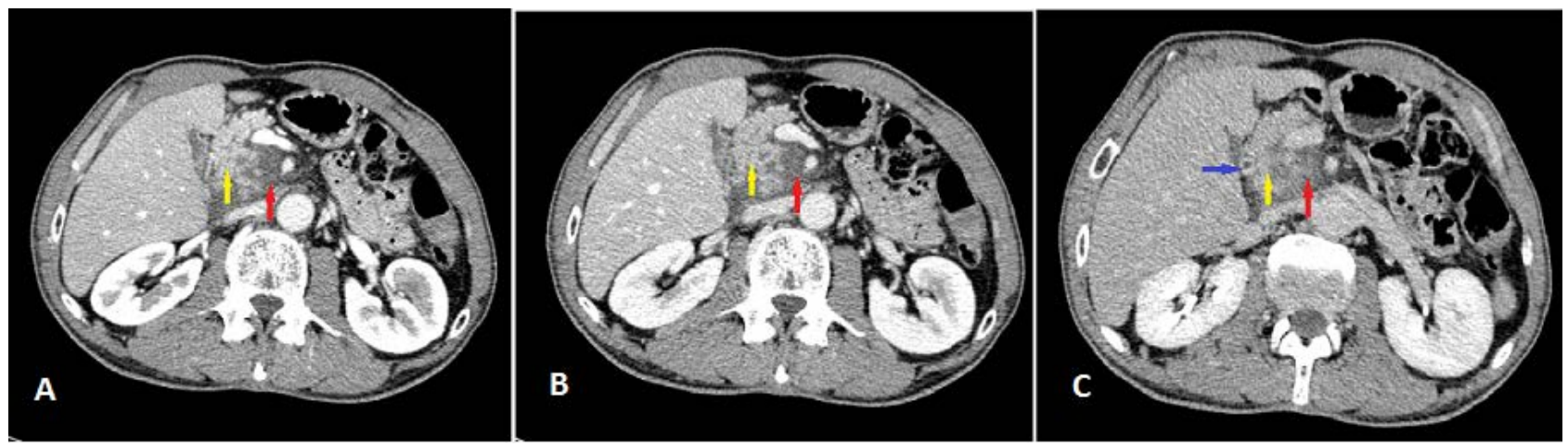

Figure 2. Axial abdominal computed tomography (CT).arterial phase (A), portal phase (B) and delayed phase (C): localized swelling of the head of the pancreas with fair enhancement resulting almost isodense in the delayed phase (yellow arrow). Hypodense peripancreatic Rim slightly enhanced in the delayed phase (red arrow).Mild dilation of common bile duct with a thickened enhanced wall (blue arrow).

contrast-enhanced MR (Figure 3). At magnetic resonance cholangiopancreatography: the main pancreatic duct shows a segmental stenosis without upstream dilation. The intrahepatic bile ducts present irregular slightly stenotic portions (Figure 4). The patient was suspected of suffering from AIP. The serum IgG levels was performed however IgG4 levels were not elevated. After multidisciplinary consultation, endoscopic ultrasonography with histological examination was judged not necessary and the patient was diagnosed with type 1 AIP according to the International Consensus Diagnostic Criteria (ICDC). Steroid therapy with oral prednisone ( $40 \mathrm{mg}$ per day) was administered for 4 weeks then it was tapered off every week by $5 \mathrm{mg}$. patient clinical conditions and laboratory tests improved. Six month later, the repeat CT scan did not evidence pancreatic 
lesions (Figure 5). The patient was followed up at the hospital and discharged after approximately 1 year.

\section{Discussion}

In 2011 the International Association of Pancreatology proposed the ICDC for
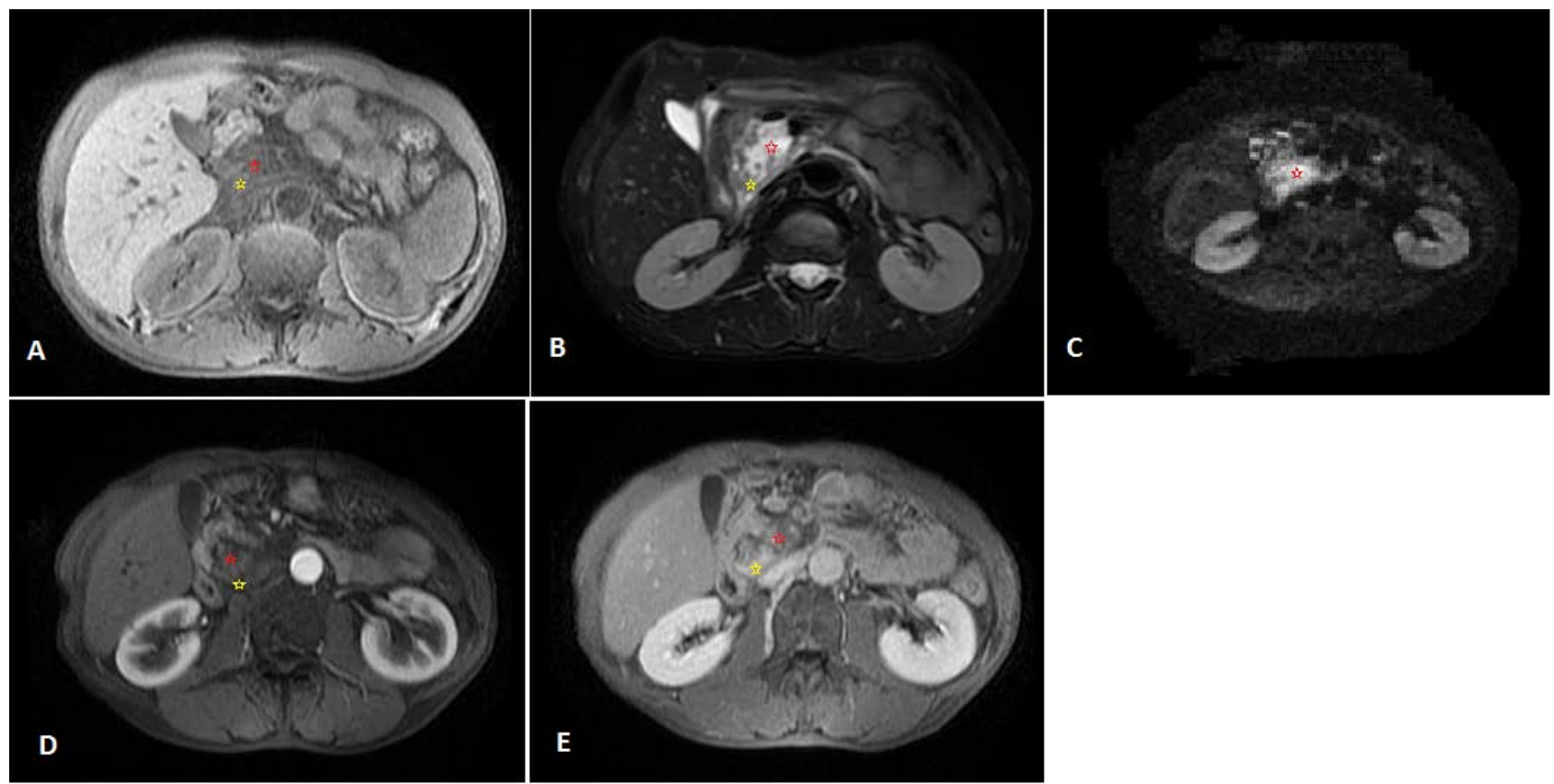

Figure 3. Magnetic resonance (MR): the affected portion of the pancreas (red asterisk) was hypointense on T1-weighted fat-saturated images (A) and hyperintense on T2-weighted fat-saturated images (B), with diffusion coefficient restriction (C). At dynamic examination, it showed heterogeneous diminished enhancement in the early phase (D) and delayed enhancement in the late phase of contrast (E). The capsule-like rim (yellow asterisk) was hypointense on both T1 (A) and T2-weighted images (B) and has delayed moderate enhancement on contrast-enhanced MR (E).
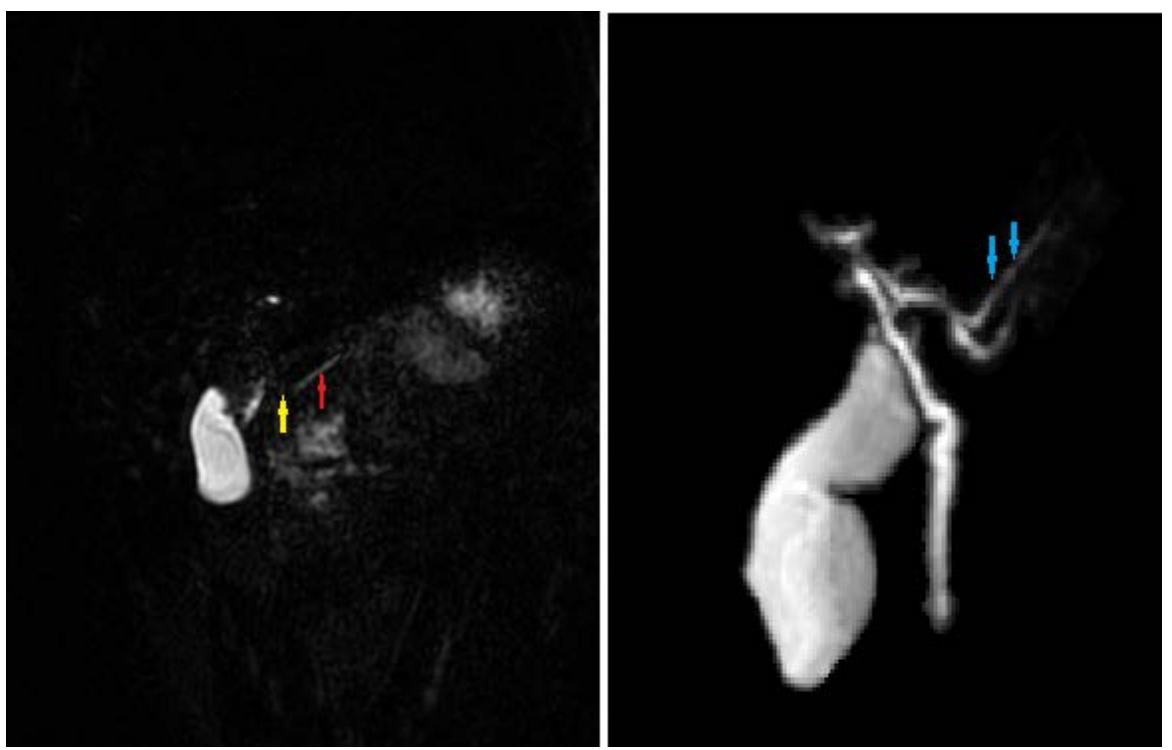

Figure 4. Magnetic resonance cholangiopancreatography (MRCP): the main pancreatic duct shows a segmental stenosis (yellow arrow) without upstream dilation (red arrow). The intrahepatic bile ducts present irregular slightly stenotic portions (blue arrow). 


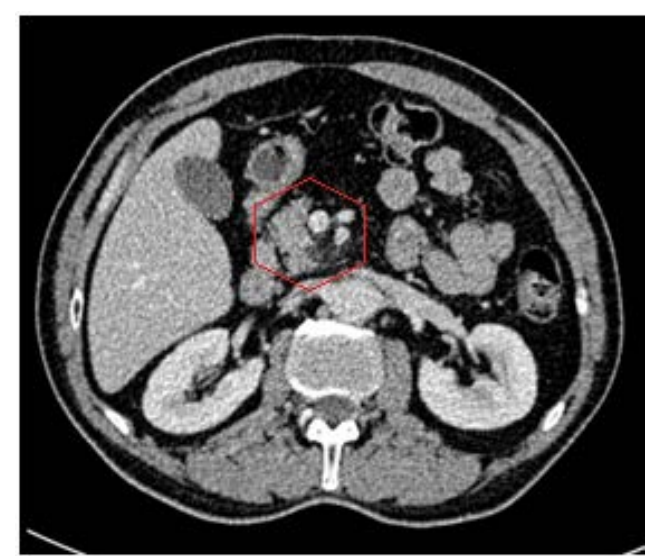

Figure 5. Control enhanced CT: a dramatic decrease in the pancreatic mass (red circle).

AIP presenting five cardinal features such as imaging of the pancreatic parenchyma on computed tomography (CT) and magnetic resonance (MR) and duct on endoscopic retrograde cholangiopancreatography (ERCP) or magnetic resonance cholangiopancreatography (MRCP),serology, other organ involvement, histology and response to steroid therapy. Each feature is categorized as a level 1 or 2 finding, depending on the diagnostic reliability. The diagnosis of type 1 and type 2 AIP can be definitive or probable [1] [2] [4].

Indeed, type 1 and type 2 AIP have distinct histopathology, clinical features and different diagnostic criteria. Type 1 AIP or lymphoplasmacytic sclerosing pancreatitis (LPSP) seems to be the pancreatic manifestation of an IgG4-related systemic disease, characterized by elevated IgG4 serum levels, infiltration of IgG4-positive plasma cells and extrapancreatic lesions (e.g., sclerosing cholangitis, sclerosing sialoadenitis and retroperitoneal fibrosis). This form of AIP presents predominantly with obstructive jaundice in elderly male subjects. Its clinical diagnosis can be made without need for a histology sample. Type 2 AIP or idiopathic duct-centric pancreatitis (IDCP) characterized by granulocyte epithelial lesions (GEL) has none or very few IgG4-positive plasma cells, no serum IgG4 elevation and appears to be a pancreas-specific disorder without extrapancreatic involvement. Approximately $30 \%$ of reported cases of IDCP are associated with inflammatory bowel disease. Patients with IDCP are, on average, a decade younger than LPSP patients and the disease does not show a sex preference. Because IDCP patients are seronegative and lack other organ involvement, definitive diagnosis requires pancreatic histology [2] [3].

AIP patients and those with PC have many clinical, biological and imaging features in common, such as elderly male's predominance, painless jaundice, weight loss, development of new-onset diabetes mellitus (DM), and elevated levels of serum tumor markers [5] [6]. Thus It is imperative to differentiate AIP from PC, especially with focal pancreatic enlargement on imaging to prevent unnecessary surgery or delayed initiation of corticosteroid therapy [1] [6].

Clinical presentation of AIP cans be acute, with obstructive jaundice by a pancreatic mass, or chronic with symptoms of chronic pancreatitis (pain, diarr- 
hea, diabetes). In AIP, jaundice can sometimes float or even spontaneously attenuate, while in PC jaundice progresses steadily.

As regards serology, a marked elevation of serum IgG4 ( $>2$ times upper normal limit) is suggestive of type 1 AIP [1]. However, we report a case of AIP compatible with type 1 AIP according to ICDC but without elevated levels of serum IgG4; it suggests that the phenotype of type 1 AIP does not require an elevation of IgG4 [7]. Other diseases such as atopic dermatitis, parasitic infections, pemphigus vulgaris, pemphigus foliaceus, and pancreatic carcinoma, can also be associated with elevated levels of IgG4 [8]. Therefore, elevation of serum IgG4 levels alone cannot rule out PC [4] and should be interpreted with caution in patients with a mass in the pancreas but no histological documentation of either carcinoma or pancreatitis [5].

Imaging plays an important role in the diagnostic work up for AIP as reflected in the different existing diagnostic criteria radiological findings [4]. We distinguish two radiological types.

The diffuse form (the most frequent, $70 \%$ of cases); the pancreas has a characteristic sausage-like appearance with diffuse enlargement and loss of the lobular contours. It is diffusely hypointense on T1-weighted images and hyperintense on T2-weighted images with diffusely enhancement on delayed phase of dynamic CT and MRI that is characteristic of AIP [4] [6] [9]. A non-enhancing fibrotic rim encircling the affected parenchyma (hypointense on both T1- and T2-weighted images) may also be present [9]. This form is rarely seen in PC [4].

The focal form (30\% of cases) manifests as a focal mass, often within the pancreatic head that may mimic a PC [6] as in our case. On CT the enlarged segment of the pancreas is typically isoattenuating or hypoattenuating to the spared, non-enlarged portion of parenchyma and may be indistinguishable from PC. At MRI the involved portion is hypointense on T1-weighted images, slightly hyperintense on T2-weighted images, and has heterogeneously diminished enhancement in the early phase and delayed enhancement in the late phase of contrast enhancement. The capsule-like rim is usually hypointense on both T1 and T2-weighted images, and has delayed moderate enhancement on contrast-enhanced MR [2]. All these features were present in our patient.

Magnetic resonance cholangiopancreatography (MRCP) has become popular as a noninvasive method for obtaining high quality images of the pancreaticobiliary tree but there has been a controversy in usefulness of MRCP in diagnosis of AIP. The major problem is that the narrowed main pancreatic duct (MPD) seen on ERCP cannot be visualized on MRCP, because of the inferior resolution of MRCP compared with ERCP [3]. However, MRCP findings of a segmental or skipped non-visualized main pancreatic duct accompanied by less upstream main pancreatic duct dilatation than what is usually seen with adenocarcinoma may suggest the presence of focal AIP [2] [3]. The MPD in focal AIP is not completely obstructed and tends to penetrate the mass after secretin administration, with the so-called "penetrating duct sign", which has been described to be highly 
specific for benign strictures. Bile duct abnormalities can be also recognized; these include smooth narrowing of the intrapancreatic portion of the common bile duct, or irregularity and stricturing of the intra- and extra-hepatic bile ducts with features similar to those seen in primary sclerosing cholangitis. Enhancing duct wall thickening is also a recognized feature and, less commonly, intra-hepatic bile duct dilation may also be observed [2] [6].

At DWI, AIP and PC are both detected as high signal intensity areas at high $b$-values images; however, PC usually presents as a solitary area, while diffuse or multiple high-intensity areas are suggestive for AIP. It has been found that mean ADC values are significantly lower in AIP than in PC, which has ADC values lower than normal pancreatic parenchyma. Muhi et al. found that the optimal ADC cutoff value ( $100 \%$ sensitivity and $89 \%$ specificity) for differentiating mass-forming AIP from PC would be $0.88 \times 10^{-3} \mathrm{~mm}^{2} / \mathrm{s}$. The reason of these findings resides in the anatomo-pathological features of these lesions: the cellularity of the dense lymphoplasmocytic infiltrate in AIP is greater than that of PC and induces lower ADC values in AIP than in PC [2].

On 18F-Fluorodeoxyglucose (FDG)-Positron Emission Tomography (PET), pancreatic FDG uptake is observed in both, but abnormal extrapancreatic uptake, such as extensive lymph nodes or swollen salivary glands, is highly suggestive of AIP [2].

Presence of other organ involvements such as bilateral salivary gland swelling, retroperitoneal fibrosis and hilar or intrahepatic sclerosing cholangitis is highly suggestive of AIP rather than PC [2] [4]. In our case, CT/MRI images demonstrated enhancement and wall thickening of the common bile duct, which might be caused by obstruction due to the enlarged pancreas head, or probably manifested as the extrapancreatic involvement in AIP associated with intrahepatic sclerosing cholangitis seen in MRCP.

In summary, imaging features that favor focal AIP over cancer include: 1) delayed homogeneous enhancement; 2) hypo attenuating capsule-like rim; 3) the absence of distal pancreatic atrophy; 4) irregular narrowing on MRCP of the MPD; and 5) irregularity and stricturing of the intra- and extra-hepatic bile ducts. Moreover, no or minimal upstream dilatation, and the absence of metastatic disease can also be helpful to differentiate AIP from PC [6] [9].

In some cases, when diagnosis is difficult histopathological examination is necessary. Endoscopic ultrasound-guided fine needle aspiration (EUS-FNA) is useful to either diagnose or rule out PC. However, definitive diagnosis of AIP is sometimes difficult by EUS-FNA, because of the small sample size obtained [4]. Our patient did not need histological evidence, two of level 1 radiological criteria was sufficient to diagnose a definitive type 1 AIP according to ICDC: a segmental narrowing of MPD without upstream dilation and stenotic portions of intrahepatic bile ducts.

A peculiar feature of AIP is the response to steroid treatment; however, corticosteroid diagnostic therapy is not generally recommended, and it should only 
be performed in carefully selected patients after obtaining negative results from a work-up for pancreatic cancer, including EUS-FNA [1]. Response to steroids is based on objective data such as radiologic evidence a dramatic decrease in the pancreatic mass or other organ involvement, resolution of the obstructive jaundice without biliary stenting, and normalization of liver function tests. If there is no such improvement or if the cancer antigen 19.9 level is rising, then the diagnosis of AIP should be reconsidered [2]. Our patient has responded dramatically to the steroid therapy both clinically and radiologically, confirming the diagnosis of type 1 AIP.

\section{Conclusion}

The case proposed demonstrates that type 1 AIP can be diagnosed without elevated levels of serum IgG4 or need for histology sample according to the ICDC, and shows how clinical and radiological presentation of AIP can be similar to that of PC especially when manifesting as a focal disease in the pancreas. Thus, AIP should be considered among differential diagnosis of PC in order to prevent unnecessary surgery or delayed initiation of corticosteroid therapy. Imaging plays an important role in the diagnostic work for AIP and can guide to benign or malign etiologies. However, radiological findings must be interpreted in clinical and analytical context.

\section{Conflict of Interest}

On behalf of all authors, the corresponding author states that is no conflict of interest.

\section{References}

[1] Franchello, A., Gonella, F., Campra, D., Limerutti, G., Bruno, M., De Angelis, C., et al. (2014) A Rare Case of IgG4-Related Systemic Disease Manifesting with Pancreatic Head Mass Mimicking Borderline Resectable Cancer. International Journal of Surgery Case Reports, 5, 1095-1097. https://doi.org/10.1016/j.ijscr.2014.11.014

[2] Crosara, S., D’Onofrio, M., De Robertis, R., Demozzi, E., Canestrini, S., Zamboni, G. and Pozzi Mucelli, R. (2014) Autoimmune Pancreatitis: Multimodality Non-Invasive Imaging Diagnosis. World Journal of Gastroenterology, 20, 16881-16890. https://doi.org/10.3748/wig.v20.i45.16881

[3] Li, Y.Q. and Xiong, X.J. (2017) Clinically Mimicking Pancreatic and Dilation in Main Pancreatic Duct in Autoimmune Pancreatitis (AIP): A Case Report and Literature Review. Open Access Library Journal, 4, e3065.

[4] Takuma, K., Kamisawa, T., Gopalakrishna, R., Hara, S., Tabata, T., Inaba, Y., Egawa, N. and Igarashi, Y. (2012) Strategy to Differentiate Autoimmune Pancreatitis from Pancreas Cancer. World Journal of Gastroenterology, 18, 1015-1020.

[5] Kamisawa, T., Chen, P.Y., Tu, Y., Nakajima, H., Egawa, N., Tsuruta, K., Okamoto, A. and Hishima, T. (2006) Pancreatic Cancer with a High Serum IgG4 Concentration. World Journal of Gastroenterology, 12, 6225-6228. http://www.wjgnet.com/1007-9327/12/6225.asp

[6] Sun, G.F., Zuo, C.J., Shao, C.W., Wang, J.H. and Zhang, J. (2013) Focal Autoim- 
mune Pancreatitis: Radiological Characteristics Help to Distinguish from Pancreatic Cancer. World Journal of Gastroenterology, 19, 3634-3641.

https://doi.org/10.3748/wjg.v19.i23.3634

[7] Nakano, E., Kanno, A., Masamune, A., Yoshida, N., Hongo, S., Miura, S., Takikawa, T., Hamada, S., Kume, K., Kikuta, K., Hirota, M., Nakayama, K., Fujishima, F. and Shimosegawa, T. (2015) IgG4-Unrelated Type 1 Autoimmune Pancreatitis. World Journal of Gastroenterology, 21, 9808-9816.

https://doi.org/10.3748/wjg.v21.i33.9808

[8] Levy, M.J., Wiesema, M.J. and Chari, S.T. (2006) Chronic Pancreatitis: Focal Pancreatitis or Cancer? Is There a Role for FNA/Biopsy? Autoimmune Pancreatitis. Endoscopy, 38, 30-35. https://doi.org/10.1055/s-2006-946648

[9] Lalwani, N., Mannelli, L., Ganeshan, D.M., Shanbhogue, A.K., Dighe, M.K., Tiwari, H.A., et al. (2015) Uncommon Pancreatic Tumors and Pseudotumors. Abdominal Imaging, 40, 167-180. 